\title{
Cyclic Path Planning of Hyper-redundant Manipulator using Whale Optimization Algorithm
}

\author{
Affiani Machmudah ${ }^{1 *}$, Setyamartana Parman², Aijaz Abbasi ${ }^{3}$, Mahmud Iwan Solihin ${ }^{4}$ \\ Teh Sabariah Abd Manan ${ }^{5}$, Salmia Beddu ${ }^{6}$, Amiruddin Ahmad ${ }^{7}$, Nadiah Wan Rasdi ${ }^{8}$ \\ Industrial Engineering, Faculty of Advance Technology and Multidisciplinary, Universitas Airlangga, Kampus C Jalan \\ Mulyorejo, Surabaya, Indonesia ${ }^{1}$ \\ Fakulti Teknologi Kejuruteraan Mekanikal dan Pembuatan, Universiti Teknikal Malaysia Melaka, 75450 Ayer Keroh, Melaka, \\ Malaysia $^{2}$ \\ Mechanical Engineering, Quaid-e-Awam University of Engineering, Science, and Technology, Nawabshah, Pakistan ${ }^{3}$ \\ Faculty of Engineering, Technology and Built Environment, UCSI University, Kuala Lumpur 56000, Malaysia ${ }^{4}$ \\ Institute of Tropical Biodiversity and Sustainable Development, Universiti Malaysia Terengganu, 21030 Kuala Nerus, \\ Terengganu Darul Iman, Malaysia, ${ }^{5,7}$ \\ Department of Civil Engineering, Universiti Tenaga Nasional, Jalan Ikram-Uniten, 43000, Kajang, Selangor Darul Ehsan, \\ Malaysia $^{6}$ \\ Faculty of Science and Marine Environment, Universiti Malaysia Terengganu, 21030 Kuala Nerus, Terengganu Darul Iman, \\ Malaysia $^{7}$ \\ Faculty of Fisheries and Food Science, Universiti Malaysia Terengganu, 21030 Kuala Nerus, Terengganu Darul Iman, Malaysia ${ }^{8}$
}

\begin{abstract}
This paper develops a path planning algorithm of hyper-redundant manipulators to achieve a cyclic property. The basic idea is based on a geometrical analysis of a 3-link planar series manipulator in which there is an orientation angle boundary of a prescribed path. To achieve the repetitive behavior, for hyper-redundant manipulators consisting of 3-link components, an additional path is chosen in such away so that it is a repetitive curve which has the same curve frequency with the prescribed end-effector path. To solve the redundancy resolution, meta-heuristic optimizations, namely Genetic Algorithm (GA) and Whale Optimization Algorithm (WOA), are applied to search optimal trajectories inside local orientation angle boundaries. Results show that using constant of the local orientation angle trajectories for the 3 -link component, the cyclic properties can be achieved. The performance of the WOA shows very promising result where generally it obtains the lowest fitness value as compare with the GA. Depending on the complexity of the path planning, dividing the path into several stages via intermediate points may be necessary to achieve the good posture. The performance of the swarm based meta-heuristic optimization, namely the WOA, shows very promising result where generally it obtains the lowest fitness value as compare with the GA. Using the developed approach, not only the cyclic property is obtained but also the optimal movement of the hyperredundant manipulator is achieved.
\end{abstract}

Keywords-Hyper-redundant; path planning; whale optimization algorithm; sustainable manufacturing

\section{INTRODUCTION}

Sustainability becomes an important achievement in all aspect of an engineering design, including in the robotics field. Optimal motion has been remarked as important goal in the sustainability design of the robotics system [1-3]. Ecoprogramming refers to approach to improve the energy efficiency on the existing plants with minimum investment cost [1]. In the robotics applications, eco-programming leverage on the developing the motion planning to reduce the energy consumption. The eco-programming can be classified to two types which are the trajectory optimization and the scheduling optimization. In the trajectory optimization, it is necessary to generate the optimal path and/or the motion profile. One of strategies to achieve the energy efficiency is by minimizing the robot motion [2]. The configuration of the robots is in the state of the most energy-efficient when the end-effector path is predefined and the path of the robot has been optimized [3].

This paper addresses the path optimization of the hyperredundant manipulator. The hyper-redundant manipulator involves the high degree of redundancy so that it has many advantages regarding their capability to avoid obstacles and achieve complex form of locomotion and grasping [4]. Because of these potential benefits, recently the hyper-redundant manipulator has been widely applied not only in the manufacturing job [5] also in challenging environments such as in the medical application [6] and the operation for rescue duty during the natural disaster [7].

The Jacobian based approach is the standard method to solve the Inverse Kinematic (IK) for the hyper-redundant manipulator [7]; however, this approach requires the matrix inversion which becomes computationally expensive for the hyper-redundant manipulator since it involves very large Degree of Freedom (DOF) [4, 8]. For the hyper-redundant case, alternative approaches have been proposed to solve the path planning problem. Ayten et al. [5] developed two methods for trajectory optimization of redundant/hyper-redundant manipulator. In their first method, the kinematic and dynamic constraints were solved sequentially according to the cost function to avoid the inverse dynamics computation. The second method used a virtual link concept replacing all the redundant link for eliminating impossible configuration before the inverse dynamic model was employed. Simulations were

*Corresponding Author 
conducted using the 3-link and 8-DOF planar series hyperredundant manipulators. Ananthanarayanan and Ordinez [9] proposed a multi-pass sequential localized search technique to solve the path planning of the hyper-redundant manipulator in the presence of obstacles. The problem was modeled as shortest path optimization where the configuration space was searched based on the cost function which needed to be optimized. Dasgupta et al. [10] applied a variational approach to optimize the path of the hyper-redundant manipulator in high dimensional C-space. Shukla et al. [11] employed the variational principles by formulating the path planning as constrained optimization. The monotonic optimality concept was proposed to obtain the optimal path between resulting end configurations. Potential fields-based path planning of the hyper-redundant manipulator had been studied by Concur [12]. An algorithm, namely point settling algorithm was proposed. A smooth path consisting of points which were closed enough each other needed to be found using harmonic potential field. The tip of each link needs to be maintained until the goal of motion was reached. Lanteigne and Jnifene [13] proposed a biologically inspired node generator for the hyper-redundant manipulator planning using a probabilistic roadmap. Chirikjian and Burdick [14] used a continuous backbone curve approach to model the kinematic of the hyper-redundant manipulator operated in uneven solid terrain.

Among many possible approaches to solve the path planning of the hyper-redundant manipulator, the geometrical approach is one of the most popular methods. Yahya et al. [8] developed the geometrical based approach by considering the joint angle between the adjacent links to be the same. By this approach, the singular configuration can be avoided since geometrically two or more joints were impossible to lining up each other. Menon et al. [7] proposed a novel optimization algorithm obtained from calculus variation for the motion planning of the hyper-redundant manipulator. The algorithm, which was purely geometric, computed the motion of all joints in such away so that all links avoid the obstacles. Sardana et al. [6] presented a simple geometrical approach to solve the IK of four degree of freedom in-Vivo robots when it inserted to the tool channel of the endoscope for taking a biopsy in the stomach. Chirikjian and Burdick [15] proposed strictly geometric algorithm for the hyper-redundant manipulator using a tool, namely tunnel, to avoid collision with obstacles. Differential geometry was used to derive the equation which guarantees that the manipulator sections were confined to the tunnel to avoid obstacles.

Despite many approaches have been proposed to solve the path planning of the hyper-redundant manipulator, only few papers address the methodology to achieve the cyclic property. The cyclic property is very essential in the manipulator motion since it exhibits the predictable behavior. The predictable behavior yields the system which is easier to be maintained than the system with random or unpredictable behavior [16]. It confines the motion within the robot operational area so that it is safe for the robot. Marcos et al. [17] proposed the combination of the GA and closed-loop pseudoinverse. The performance of the Open Loop GA (OLGA) and Closed Loop GA (CLGA) were compared. Result showed that CLGA had good performance than that of the CLGA where the CLGA yielded the repetitive behavior. However, using the CLGA approach, the matrix inversion was still necessary. The fractional calculus had been studied to solve the hyperredundant path planning problem [18, 19]. This approach needed to find the value of $\alpha$ in order to get the cyclic joint angle trajectories.

This paper develops the path planning approach of the $n$ link planar series hyper-redundant manipulator to achieve the cyclic properties based on the geometrical approach incorporating the meta-heuristic optimization. The hyperredundant manipulator is modeled as composition of the 2-link and 3-link components. The last 3-link component tracks the end-effector path with respect to position of the previous link as the moving base. The constant parameter, $k$, which gives the optimal path needs to be searched within this boundary employing the meta-heuristic optimization, namely the GA and WOA. As an advantage, since the geometrical approach is employed, the proposed approach does not need the matrix inversion. Furthermore, both the optimization criteria which is the minimum joint angle path and the cyclic property which yields the predictable behavior can be achieved.

The presentation of the paper is in the following. The path planning optimization is presented in Section 2. Section 3 presents the proposed path planning method. Joint angle trajectories generation is described and the algorithm to achieve the cyclic property for the hyper-redundant planar series manipulator is presented. Section 4 presents the metaheuristic optimizations, which are the GA and the WOA. Section 5 presents numerical experiments of the path planning of the 5-DOF and 12-DOF planar series hyper-redundant manipulator.

\section{PAth PlanNing OPtimizations}

The path planning is modeled as the constraint optimization problem with the optimization objective is to minimize the joint angle path:

$\operatorname{Min} F_{p a t h}=\sum_{i=1}^{n} \int_{0}^{1} \sqrt{1+\left(\frac{d \theta_{i}(r)}{d r}\right)^{2}} d r$

Subject to:

$\theta_{\text {imin }} \leq \theta_{i} \leq \theta_{\text {imax }}$

$x=l_{1} \cos \left(\theta_{1}\right)+\ldots . l_{i} \cos \left(\theta_{1}+\theta_{2}+\ldots \theta_{i}\right)$

$y=l_{1} \sin \left(\theta_{1}\right)+\ldots l_{i} \sin \left(\theta_{1}+\theta_{2}+\ldots \theta_{i}\right)$

$x_{e}=f(t) \quad ; \quad y_{e}=g(t)$

$\left(x_{e}, y_{e}\right)=(x, y)$

$\left(\left(x_{\text {link }}, y_{\text {link }}\right) \cap o b s\right)=\emptyset$

where $\left(x_{\mathrm{e}}, y_{\mathrm{e}}\right)$ and $(x, x)$ are desired end-effector position and actual end-effector position, $\theta_{i}$ is a joint angle of ith link, $l_{i}$ is length of $i$ th link, $\theta_{i \min / \max }$ is minimum/maximum $i$ th joint angle, $\left(x_{\text {link, }}, y_{\text {link }}\right)$ and obs are link configurations and obstacle area, with $n$ is number of links, respectively.

Equation (2) is the constraint of the joint angle. Equations $(31,3 b)$ are the forward kinematics of the planar series hyperredundant manipulator. 


\section{PRoposed PATH PLANNING}

For the IK problem of 3-DOF planar series manipulator, it has the analytic solution using the geometrical approach in the following [20]:

$w_{x}=x_{p}-l_{1} \cos \left(\theta_{g}\right) ; w_{y}=y_{p}-l_{1} \sin \left(\theta_{g}\right)$

$c_{2}=\frac{\left(w_{x}{ }^{2}+w_{y}{ }^{2}-l_{1}{ }^{2}-l_{2}{ }^{2}\right)}{2 l_{1} l_{2}}$

$s_{2}= \pm \sqrt{1-c_{2}^{2}}$

$\theta_{2}=\operatorname{atan} 2\left(s_{2}, c_{2}\right)$

Second and third joint angles can be obtained by following equations:

$\Delta=w_{x}^{2}+w_{y}{ }^{2} ; S_{1}=\frac{\left(l_{1}+l_{2} c_{2}\right) w_{y}-\left(l_{2} s_{2} w_{x}\right)}{\Delta}$

$c_{1}=\frac{\left(l_{1}+l_{2} c_{21}\right) w_{x}+\left(l_{2} s_{21} w_{y}\right)}{\Delta} ; \theta_{1}=\operatorname{atan} 2\left(s_{1}, c_{1}\right)$

$\theta_{3}=\theta_{g}-\theta_{3}-\theta_{1}$

where $\theta_{1 s}, c_{1}, s_{1}$ and $\theta_{3 s}$ are the first joint angles, the cosine of $\theta_{1 \mathrm{~s}}$, the sine of $\theta_{1 \mathrm{~s}}$, and the third joint angle, respectively.

Equation (8a) can be expressed into more useful functions as follows:

$c_{2}=A_{x} \cos \theta_{g}+A_{y} \sin \theta_{g}+k_{p}$

$=A_{p} \cos \left(\theta_{g}-\varphi_{p}\right)+k_{p}$

$A_{x}=\frac{-l_{3} x_{p}}{l_{1} l_{2}} ; A_{y}=\frac{-l_{3} y_{p}}{l_{1} l_{2}}$

$\varphi_{p}=\operatorname{atan} 2\left(A_{y}, A_{x}\right) ; k_{p}=\frac{R^{2}+l_{3}{ }^{2}-l_{2}{ }^{2}-l_{1}{ }^{2}}{2 l_{1} l_{2}} ;$

$R=\sqrt{x_{p}^{2}+y_{p}^{2}}$

where $\phi_{p}, k_{p}, A_{p}$, and $R$ are a phase shift, a vertical shift, an amplitude, a radius from the fix base, respectively.

\section{A. Joint Angle Trajectory Generation}

This paper proposes to model the hyper-redundant manipulator as components of 2-link and 3-link.

For the 2-link component, the joint angle trajectories are modelled as the polynomial degree sixth as follows [21]:

$\theta_{k}=a_{6 k} r^{6}+a_{5 k} r^{5}+a_{4 k} r^{4}+a_{3 k} r^{3}+a_{0 k}$

$\mathrm{a}_{0 \mathrm{k}}=\theta_{\mathrm{ik}} ; a_{5 k}=-3 a_{6 k}-6 \theta_{i k}+6 \theta_{f k}$;

$a_{4 k}=0.5\left(-9 a_{6 k}-5 a_{5 k}\right)$

$a_{3 k}=\theta_{f k}-\theta_{i k}-a_{6 k}-a_{5 k}-a_{4 k} ; r=\frac{t}{T_{-}}$

where $\theta_{k}, \theta_{i k}, \theta_{f k}, a_{n k}$, and $r$, are the joint angle of $k$ th link, the joint angle of $k^{\text {th }}$ link at $t=0 \mathrm{~s}$, the joint angle of $k^{\text {th }}$ link at $t=T \_$s, $n$th polynomial coefficient of $k$ th link, and linear timescale, respectively.

$T_{-}$is the period of the repetitive curve or the time to conduct one complete end-effector repetitive curve. By designing the joint angle at initial point of repetitive curve, $t=0$ $\mathrm{s}$, to the final point of repetitive curve, $t=T_{-} \mathrm{s}$, as the same value, the periodic joint angle trajectories can be achieved. Depending on the complexity of the path planning, the intermediate points may be needed to achieve the good posture.

For the 3-link component, the joint angle trajectory is simply chosen as constant value as follows:

$\theta_{g k}(t)=k$

where $\theta_{g k}$ and $k$ are the local orientation angle boundary of $k$ th 3-link component and constant parameter, respectively.

The value of $A_{p}, k_{p}$, and $\phi_{p}$ are functions of tracked positions $\left(x_{p}, y_{p}\right)$. Since $\left(x_{p}, y_{p}\right)$ trajectories are varied, then $A_{p}$, $k_{p}$, and $\phi_{p}$ continuously changes, there will be the lower bound trajectories of , $\theta_{\text {gmin }}$, and the upper bound trajectories, $\theta_{\text {gmax }}$, of $\theta_{g k}$ constructing a boundary. Since the redundancy resolution with respect to the position of previous 2-link or 3link components has been mapped into the boundary, any arbitrary function, $F(t)$, generated inside the boundary of $\theta_{g}$ are possible solutions. The position of links can be obtained from the forward kinematics, Eq. (3).

For the 3-link component, it has the joint angle component as follows:

$\theta=\left[\begin{array}{lll}\theta_{k} & \theta_{k+1} & \theta_{k+2}\end{array}\right]$

where $\theta_{k}$ is the joint angle of $k$ th link.

Each component of $\theta$ can be determined as follows:

$\theta_{k}=\theta_{1 s}-\sum_{n=1}^{j-1} \theta_{n} ; \theta_{k+1}=\theta_{2 s} ; \theta_{k+3}=\theta_{3 s}$

where $\theta_{1 s}, \theta_{2 s}, \theta_{3 s}$ are Equation (11), Equation (9), Equation (12), respectively.

The above joint angle is computed with respect to the moving base where it can be obtained by Eqs. (7-12) through modifying equation (7) as follows:

$w_{x}=\left(x_{k}-x_{k-1}\right)-l_{k} \cos \left(\theta_{g}\right)$

$w_{y}=\left(y_{k}-y_{k-1}\right)-l_{k} \sin \left(\theta_{g}\right)$

where $l_{k}$ and $\left(x_{k}, y_{k}\right)$ are length of $k$ th link and position of the end of $k$ th link, respectively.

The local orientation angle represents the total local joint angle, $\theta_{g k}=\theta_{k}+\theta_{k+1}+\theta_{k+2}$

\section{B. Algorithm}

Fig. 1 shows the flow chart of the proposed algorithm. The proposed path planning to achieve the cyclic property of the hyper-redundant manipulator can be computed using the following procedure:

1) For the hyper-redundant robot consist of 3-link

a) Define an additional path as moving base of 3-link components. The additional path can be chosen as repetitive 
path having same curve frequency with the end-effector path. The first 3-link component has the fix base.

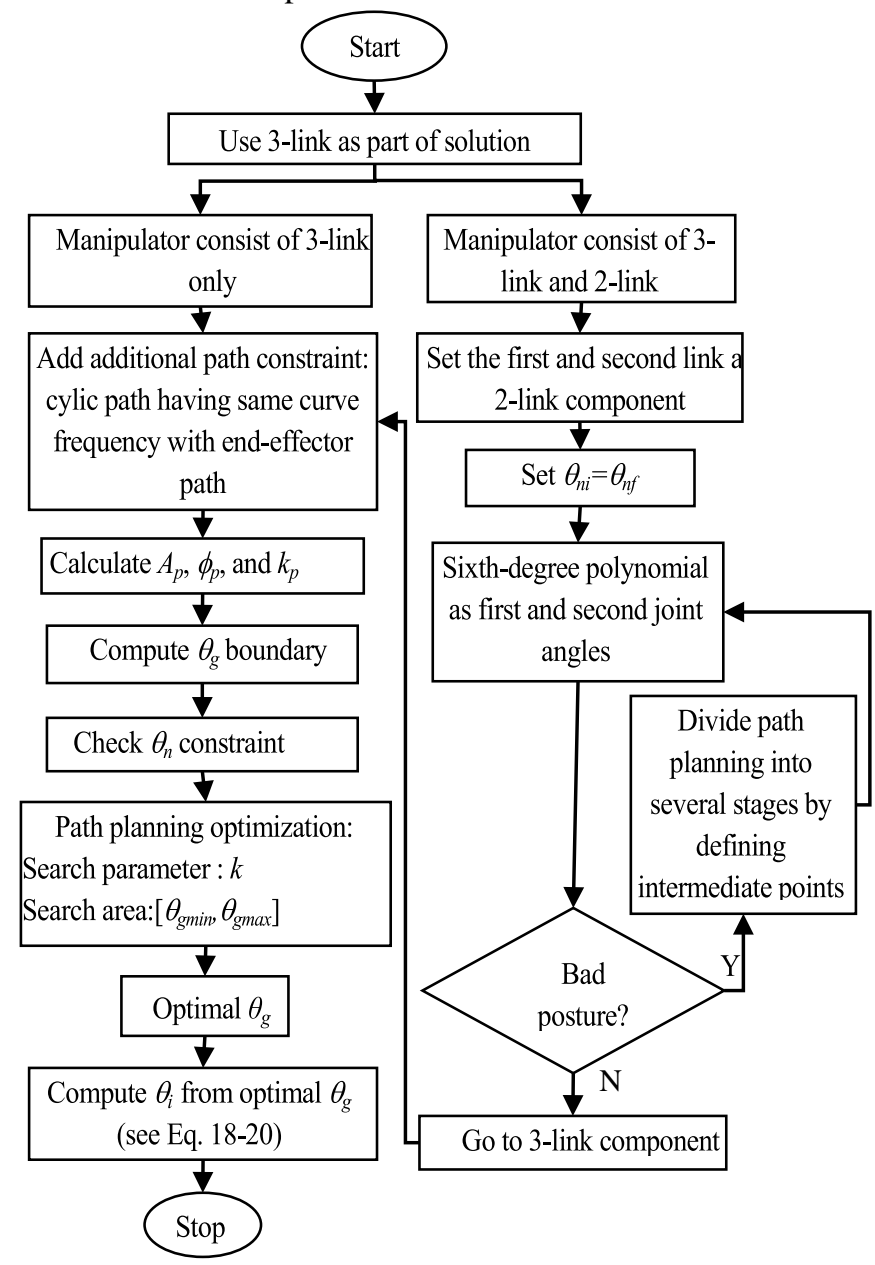

Fig. 1. Flow Chart of the Proposed Geometrical based Approach.

b) Calculate Amplitude $A_{p}$, phase shift $\phi_{\mathrm{p}}$, and the vertical shift $k_{p}$ to achieve the moving base for 3-link components. The last 3-link component tracks the end-effector path.

c) Compute lower bound $: \theta_{\text {gmin }}$ and upper bound $\theta_{g \max }$ for all sampling points.

d) Check constraint of joint angle. The boundary may decrease if there is the joint angle constraint.

e) Optimize the orientation angle trajectories, Eq. (17), using the meta-heuristic optimization.

f) Go to step 3. link

2) For the hyper-redundant robot consist of 2-link and 3-

a) Set the first and second link as 2-link component.

b) Define point A and point B inside the intersection of workspace of 2-link and 3-link components. c) Use polynomial degree sixth as the first and second joint angles. Set joint angle at initial position of the repetitive curve, $t=0$, and final position of the repetitive curve, $t=T_{-}$, as the same value to achieve cyclic property.

d) In the case of bad posture detected, divide the path planning into several stages by defining the intermediate point and do step (iii) for all stage. To achieve connectivity between the current stage and the previous stage, final joint angle of $k$ th stage become initial joint angle of $(k+1)$ th stage.

$e$ ) Calculate the position of the end of 2-link component $\left(x_{2}, y_{2}\right)$, which becomes the moving base of next 3-link component, using forward kinematics.

f) For next 3-link components, do step (a) to (d) of step 1 with respect to the moving base. The moving base for the first 3-link component is obtained from (v) result.

g) Construct the overall orientation angle boundary for one cyclic time by combining the boundary of the overall stage. The boundary should be connected between the neighboring stages.

h) Optimize the local orientation angle trajectories, Eq. (17), using the meta-heuristic optimization.

3) Compute $\theta_{\mathrm{i}}$ from optimal $\theta_{\mathrm{g}}$ trajectories using Eqs. (712) for the fixed base. For the moving base, use Eqs. (18-20).

\section{Meta-Heuristic Optimizations}

To choose the best solution among many possible solutions of the IK, the meta-heuristic optimizations, which are the GA and the WOA are employed.

\section{A. Fitness Functions}

The optimal solution with the optimization objective is to minimize joint angle traveling distance considering the constraints as follows:

$F_{\text {obj }}= \begin{cases}0 & \text { if } \exists\left(x_{\text {link }}, y_{\text {link }}\right) \cap \text { obs } \\ F_{\text {path }} & \text { else }\end{cases}$

where $F_{\text {path }}$ is equation (1).

In the case there is no obstacle, the objective function is $F_{\text {path }}$. This paper considers the intermediate points which are pre-defined, and the orientation angle trajectories of 3-link component are optimized. Thus, the parameter to be searched in the optimization is $k$ in Eq. (19). The total number of searching parameter depends on the number of links.

\section{B. Genetic Algorithm}

The GA has three main procedures which are reproduction, crossover, and mutation. The searching parameter is represented as the chromosome. A crossover is a process of randomly picking one or more individual as parents and swapping segments of the parents. The mutation is the GA part which corresponds to the search space exploration. This paper employs real code GA where random resetting mutation is used. In this scheme, randomly chosen gene assigns to be changed with a random value. 
The following is the GA pseudocode of the path planning of the hyper-redundant manipulator:

\section{Begin}

$t=0$

Initialize parameter of the GA, chromosome is $k$, equatioi (17)

Calculate $\theta$ i, equations (7-12, 18-20)

Compute the fitness, equation(21 )

Evaluate individuals in population

Store best individual

If number iteration $<=$ maxit

$t=t+1$

Create mating pool

New offspring by crossover

Mutation

Go to step 3

Output best and stop

End

\section{Whale Optimization Algorithm}

The WOA is relatively new meta-heuristic optimization technique inspired from the bubble-net hunting strategy of humpback whales proposed by [22]. There are three main important scenarios in the WOA, which are Encircling prey, bubble-net attacking (exploitation phase), and Search for prey (exploration phase).

The search agents update their positions approaching the best search agent. This behavior is expressed by:

$\vec{D}=\overrightarrow{\mathrm{C}} \overrightarrow{\mathrm{X}}^{*}(\mathrm{t})-X$

$\vec{X}(t+1)=\overrightarrow{\mathrm{X}}^{*}(\mathrm{t})-A \cdot \vec{D}$

where $t, \vec{A}, \vec{C}, \mathrm{X}^{*}, \vec{X}$, and || are the current iteration, a coefficient vector, the current position vector of the best solution, the position vector, the absolute value, and an element-by-element multiplication. $X^{*}$ needs to be updated in the computation when there is a better solution.

$\vec{A}$ and $\vec{C}$ are expressed mathematically as follows:

$\vec{A}=2 \vec{a} \cdot \vec{r}-\vec{a}$

$\vec{C}=2 \cdot \vec{r}$

$\vec{a}$ is decreased from 2 to 0 in exploration and exploitation phases and $\vec{r}$ is a random vector in $[0,1]$.
The following is the WOA pseudocode of the path planning of the hyper-redundant manipulator:

Initialize whales population $X_{i}$, with positions $=k(E q .17)$

while ( $t<$ maximum number of iterations)

Calculate $\theta$ i, Eqs. (7-12, 18-20)

Calculate the fitness of each search agent

$X^{*}=$ the best search agent

for each search agent

Update a, $A, C$

If $1(\rho<0.5)$

if $2(|A|<1)$

Update the position of the current search agent

Else if2 $(|A|>=1)$

Select a random search agent (Xrand)

Update the position of the current search agent

End if2

Else if $1(\rho>=0.5)$

Update the position of the current search

End if1

End for

Check if any search agent goes beyond the search space

and amend it

Calculate Oi, Eqs. (7-12, 18-20)

Calculate the fitness of each search agent

Update $X^{*}$ if there is a better solution

$t=t+1$

End while

Return $X^{*}$

\section{RESUlt AND DisCUSSION}

A numerical experiment has been conducted in MATLAB environment by writing a computer program. For the GA, the real value coded is used with the selection rate and the mutation rate are 0.5 and 0.1 , respectively. Both GA and WOA are evaluated using 100 numbers of iterations and 20 individuals in the population. The value of the searching parameter, $\mathrm{k}$, is set to be four decimal place accuracies.

\section{A. 5-DOF}

This section applies the proposed approach to the case 3 in [8] with the goal is to achieve the optimal path while satisfying the cyclic property. Yahya et al. [8] proposed to apply the geometrical approach for the planar 5-DOF hyper-redundant manipulator by setting the second, third, fourth, and fifth joint angles to have the same value so that the singular configuration can be avoided while the good posture can be achieved; however, they did not discuss how to achieve the cyclic property. 
The 5-DOF planar series manipulator with length, $l=[2220$ $181614] \mathrm{cm}$, tracks the curve as follows:

$$
\begin{aligned}
& x(t)=30 \cos (t+3)-25 \\
& y(t)=30 \sin (t+3)+30
\end{aligned}
$$

The above curve has period $T_{-}=2 \pi$ second. Using the proposed approach, the moving base, which is the position of the end of second link $\left(x_{2}, y_{2}\right)$, should be kept inside the workspace of the 3-link components. Fig. 2a shows the local orientation angle boundary using one step path planning with initial/final first joint angle: $\theta_{1 i}=\theta_{2 i}=-0.8$ and initial/final second joint angle: $\theta_{1 f}=\theta_{2 f}=0.8$. Using the value of $a_{6 k}=10$ and $\theta_{g k}=3$, Fig. 2b shows the posture to track the above curve. It can be observed that there are bad postures involved.

To achieve the good posture, the proposed path planning is applied by dividing the path planning step into four stage every period time as follows:

$$
r(t)=\left\{\begin{array}{cc}
\frac{2}{\pi} t & d T_{-} \leq \mathrm{t} \leq \frac{T}{4} d T_{-} \\
t-\frac{2}{\pi} & \left(\frac{T_{-}}{4} d T_{-}+d T_{-}\right) \leq \mathrm{t} \leq\left(\frac{T_{-}}{2}+d T_{-}\right) \\
\frac{2}{\pi}(t-\pi) & \left(\frac{T_{-}}{2}+d T_{-}\right) \leq \mathrm{t} \leq\left(\frac{3 T_{-}}{4}+d T_{-}\right) \\
\frac{2}{\pi}\left(t-\frac{3 \pi}{2}\right) & \left(\frac{3 T_{-}}{4}+d T_{-}\right) \leq \mathrm{t} \leq\left(T_{-}+d T_{-}\right)
\end{array}\right.
$$

where $r, T_{-}$, and $d$ are the linear time-scale, period, and the whole number, respectively.

For the first 2-link component, at each stage, the polynomial degree sixth, Eq. (16), is generated with the definition of $r$ as Eq. (25). Using this definition, every stage is computed from parameter 0 to 1 . The initial joint angle and final joint angle of each stage for the first and second links are presented in Table I.

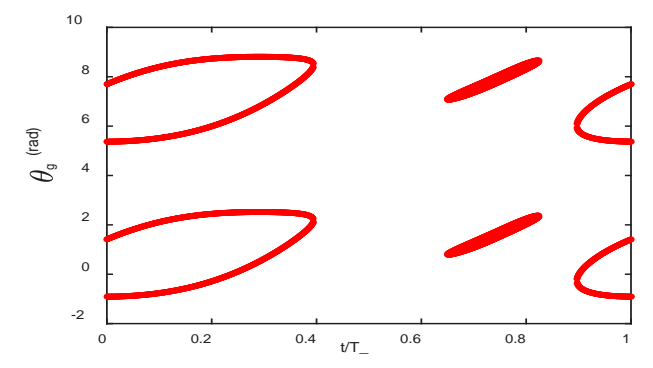

(a)

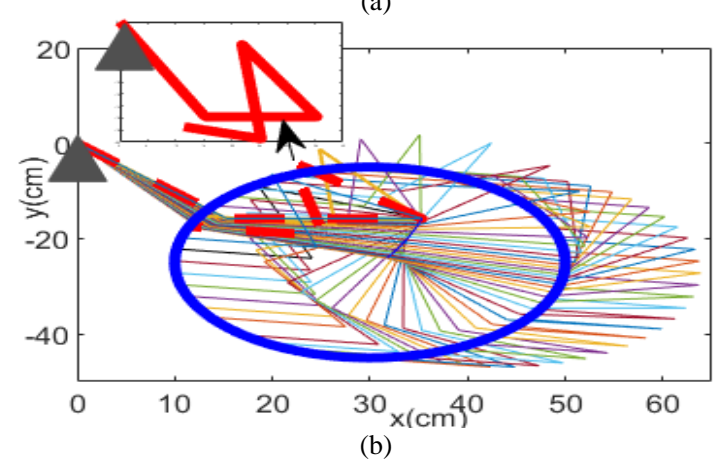

Fig. 2. One Time Path Planning (a) Boundary (b) Containing Bad Posture.
TABLE I. INITIAL AND FINAL ANGLES OF FIRST AND SECOND JoINT

\begin{tabular}{|l|l|l|l|l|l|}
\hline \multicolumn{2}{|l|}{ Joint(rad) } & I & II & III & IV \\
\hline \multirow{2}{*}{1 st } & Initial & -2.75 & -2.6 & -1.8 & -2 \\
\cline { 2 - 6 } & final & -2.6 & -1.8 & -2 & -2.75 \\
\hline \multirow{2}{*}{ 2nd } & Initial & 1.3 & 1.8 & 1 & 2 \\
\cline { 2 - 6 } & final & 1.8 & 1 & 2 & 1.3 \\
\hline
\end{tabular}

The final joint angle of kth stage becomes the initial joint angle of $(k+1)$ th stage to achieve connectivity between the current stage and the previous stage. The position of the end of second link $\left(x_{2}, y_{2}\right)$ is obtained from the forward kinematics. Using $a_{6 k}=0$, with respect to this second link position which become the moving base of the 3-link component, the local orientation angle boundary is illustrated in Fig. 3.

The local orientation angle trajectories, $\theta_{g k}(t)$, should be kept inside the angle domain boundary. This paper considers the constant trajectories as Eq. (17). The optimum value of $\mathrm{k}$ needs to be searched inside the local orientation angle boundary. The geometrical approach of the 3-link component has two possible postures obtained from the positive and negative roots of Eq. (8b). The optimal configuration come from the both possible postures is searched using the GA and the WOA. Table II presents the path planning results of the GA and the WOA for both possible postures. Fig. 4a and 4b shows the fitness value evolution for the GA and the WOA for the positive root and the negative root, respectively. For both cases, it shows that the WOA has lowest fitness value as compare with the GA results.

Using the optimum value of $\mathrm{k}$ from the WOA, the joint angle trajectories for m-cycle of end-effector movement from the positive and negative roots are illustrated in Fig. 5 and 6, respectively. It can be observed that the joint angle come from the both possible postures are periodic so that they are satisfy the cyclic property. Fig. 7a and 7b illustrate the postures for each stage of the path planning and overall stage of one cycle of end-effector movement, respectively, for the positive root. Fig. 8a and 8b show the postures for each stage of the path planning and overall stage of one cycle of end-effector movement, respectively, for the negative root.

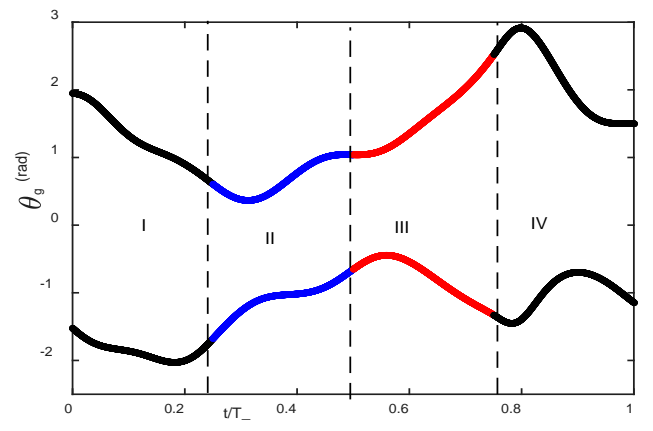

Fig. 3. Boundary of 4-Stage Path Planning.

TABLE II. PAth Planning Results

\begin{tabular}{|l|l|l|}
\hline Methods & Fitness & $\mathrm{k}$ \\
\hline GA & 20.2871801 & -0.2838 \\
\hline WOA & 20.2871779 & -0.2828 \\
\hline
\end{tabular}




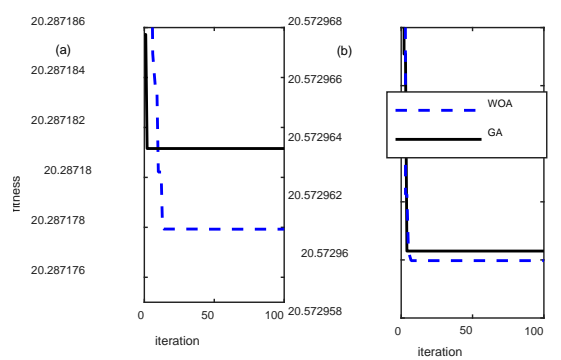

Fig. 4. Fitness Value Evolution (a) $+\sqrt{1-\mathrm{c}_{2}{ }^{2}}(\mathrm{~b})-\sqrt{1-\mathrm{c}_{2}{ }^{2}}$.

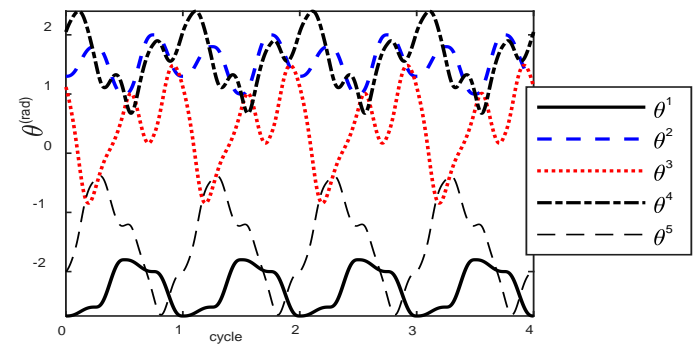

Fig. 5. Joint Angle for M-Number of Cycles, $+\sqrt{1-\mathrm{c}_{2}}$.

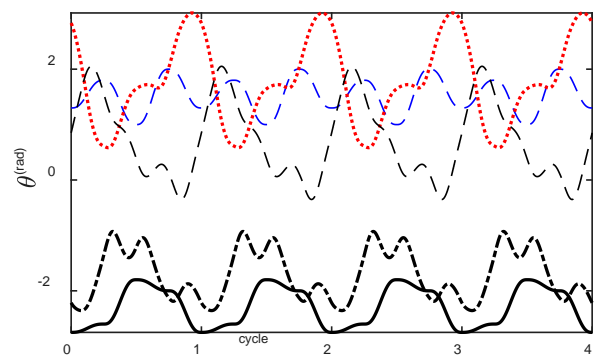

Fig. 6. Joint Angle for M-Number of Cycles, $-\sqrt{1-\mathrm{c}_{2}{ }^{2}}$.

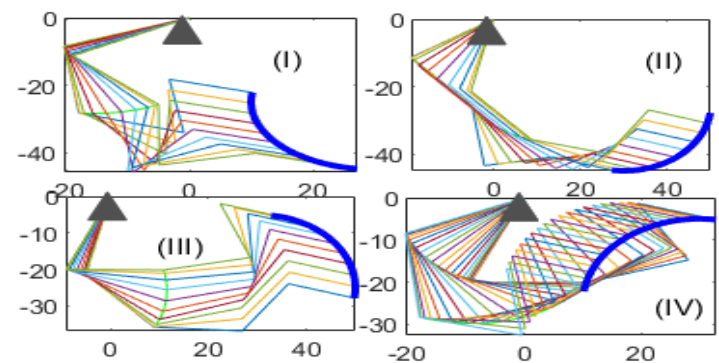

(a)

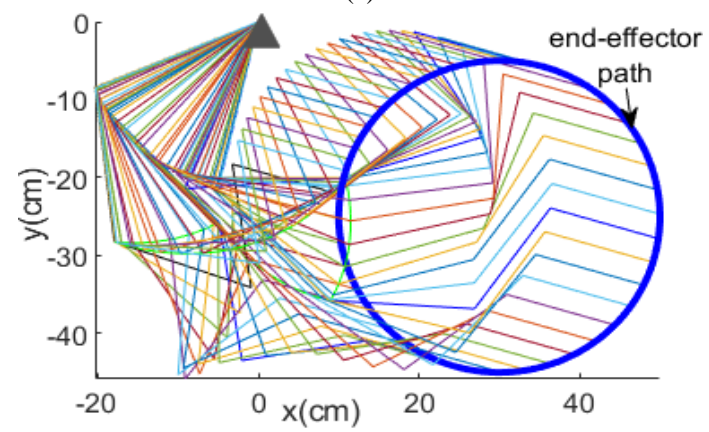

(b)

Fig. 7. Posture $=+\sqrt{1-c_{2}^{2}}$ (a) Each Stage (b) Overall Stage.
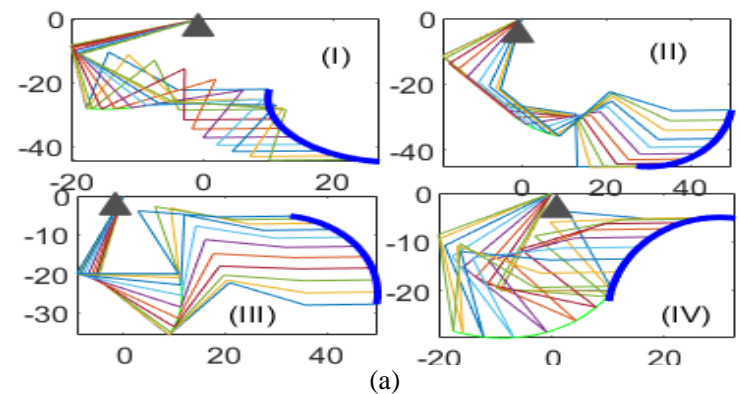

(a)

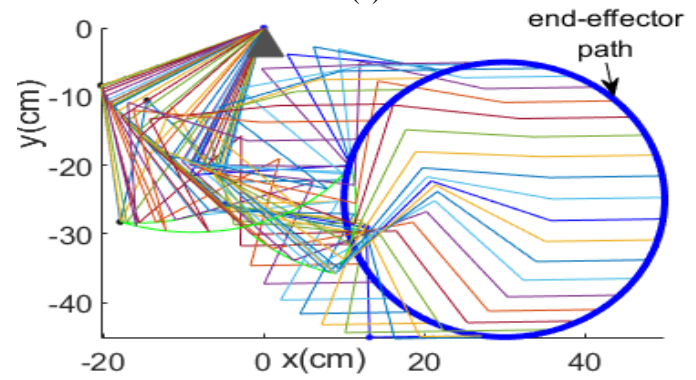

(b)

Fig. 8. Posture $=-\sqrt{1-c_{2}^{2}}$ (a) Each Stage (b) Overall Stage.

It can be observed that both possible postures do not contain any bad posture as when the path planning is conducted in one step path planning without the intermediate point (see Fig. 2b).

\section{B. 12-DOF Planar Series Manipulator}

This section studies the application of the proposed method to a 12-DOF planar series hyper-redundant manipulator. The manipulator has the same link length, $30 \mathrm{~cm}$.

The desired end-effector path is a closed planar curve, namely a Japanese maple leaf which can be described in polar coordinate as follows:

$\rho=k[(1+\sin \Omega t)(1+0.3 \cos 8 \Omega t)(1+0.1 \cos 24 \Omega t)]$

$\Omega=\frac{2 \pi}{T_{-}}$

where $\Omega$ and $k$ are the angular frequency and scale factor, respectively.

This paper considers the joint angle constraint as follows:

$0 \leq \theta_{2} \leq \pi ; 0 \leq \theta_{5} \leq \pi ; 0 \leq \theta_{8} \leq \pi$

$0 \leq \theta_{11} \leq \pi$

For the hyper-redundant consists of only 3-link components, the first 3-link component, which has fixed base, should follow the first moving base. This first moving base become the base of the second 3-link component which should follow the second moving base. This mechanism is continued until the last 3-link component tracks the prescribed endeffector path. According to this scenario, for 12-DOF hyperredundant manipulator, there are four local orientation angle boundaries. These boundaries represent the redundancy resolutions to track the desired end-effector path. The moving base is designed as the Lissajous curve with different parameter (see Table III). 
TABLE III. MOVING BASE DEFINITION

\begin{tabular}{|l|l|l|}
\hline Moving base & Curve & Search area \\
\hline \multirow{2}{*}{ I } & $x(t)=12 \sin (2 \Omega t)+40$ & \multirow{2}{*}[-0.4,0.9]{} \\
\cline { 2 - 2 } & $y(t)=12 \sin (\Omega t)+30$ & \\
\hline \multirow{2}{*}{ II } & $x(t)=12 \sin (\Omega t)+100$ & {$[0,0.7]$} \\
\cline { 2 - 2 } & $y(t)=12 \sin (2 \Omega t+\pi / 6)+70$ & \multirow{2}{*}[-1.6,-0.9]{} \\
\hline \multirow{2}{*}{ III } & $x(t)=12 \sin (\Omega t)+140$ & \\
\cline { 2 - 3 } & $(t)=12 \sin (2 \Omega t)+10$ & \\
\hline
\end{tabular}

There are three obstacles which have circular geometry with radius $10 \mathrm{~cm}$. The positions of obstacles are $(95,15)$, $(145,65)$, and $(40,-15)$. Due to the obstacles, the searching area of the first, second, and third 3-link components is reduced as described in Table III. For the fourth 3-link component, the searching area is $[0.9,1.6]$. Because of the joint angle constraint, Eq. (27), only positive root of Eq. (8) are satisfied. Each joint angle trajectories of the 3-link component should be optimized with the detail results of the GA and the WOA presented in Table IV.

TABLE IV. Path Planning Results of Japanese Maple Leaf CuRVE PATH BY 12-DOF MANIPULATOR

\begin{tabular}{|l|l|l|l|l|}
\hline \multirow{2}{*}{$\begin{array}{l}\text { 3- } \\
\text { link }\end{array}$} & GA & WOA & \\
\cline { 2 - 5 } & Fitness & $\boldsymbol{k}$ & Fitness & $\boldsymbol{k}$ \\
\hline 1 & 8.520017 & -0.2964 & 8.520015 & -0.2959 \\
\hline 2 & 10.7813247 & 0.2857 & 10.7813234 & 0.2861 \\
\hline 3 & 5.1071097 & -1.3635 & 5.1071078 & -1.3644 \\
\hline 4 & 15.25502 & 1.4053 & 15.255025 & 1.4053 \\
\hline
\end{tabular}

Fig. 9 illustrates the fitness evolution of the GA and WOA for each 3-link component. It shows that the WOA has outperformed the GA for the first, second, and third 3-link components where the WOA yield the lowest fitness value. For the fourth 3-link component, the GA and WOA yield the same optimal value.

Fig. 10 shows the joint angle trajectories for each 3-link component for m-cycle of curve. These joint angle trajectories are repeated every period time. It can be observed that the trajectories are periodic so that the proposed solution exhibits the cyclic properties. Fig. 11 shows the optimal posture of the hyper-redundant robot to track the Japanese maple leaf curve using the optimal $\mathrm{k}$ result obtain from WOA.
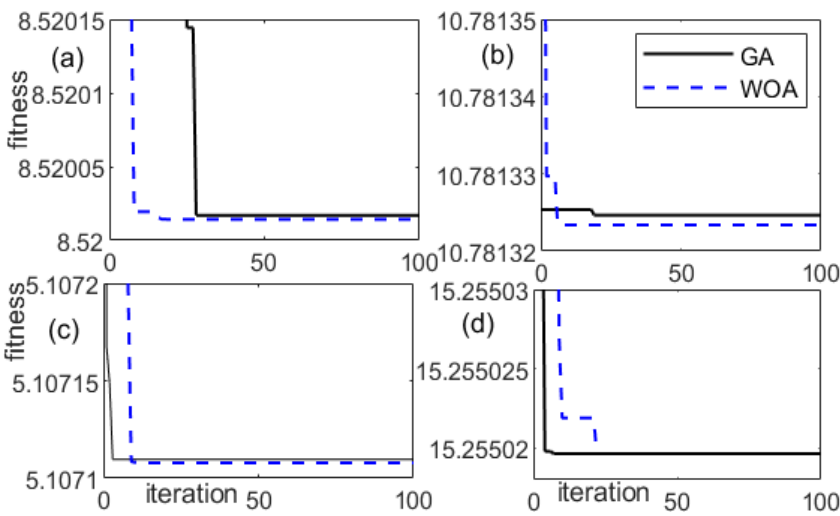

Fig. 9. Fitness Value Evolution of GA/ WOA of 3-Link Component (a) 1st (b)2nd (c) 3rd (d) $4^{\text {th }}$.
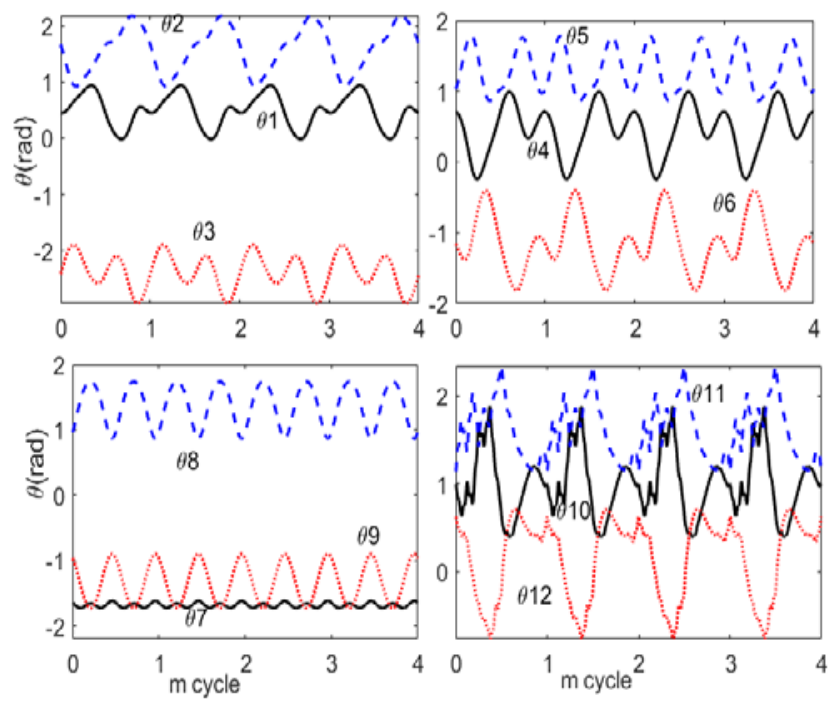

Fig. 10. Optimal Results of Joint Angles.

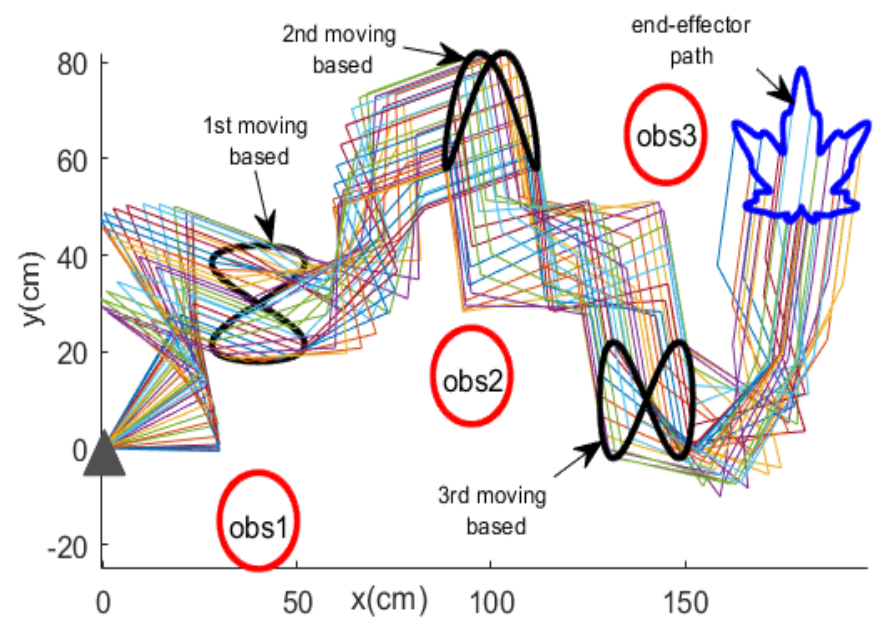

Fig. 11. Optimal Posture.

\section{Comparison with Pseudoinverse Jacobian}

The Jacobian-based method becomes the standard method to solve the IK of the hyper-redundant manipulator. This section investigates the performance of the Pseudoinverse Jacobian in solving the motion planning of the 12 DOF planar series manipulator presented in the previous sub-section.

Fig. 12 shows the joint angle trajectories and postures of the 12-link planar manipulator for 500-cycle of motion. The joint angle trajectories obtain by employing Pseudoinverse Jacobian with initial configurations are the same as starting configuration in the previous simulations. It shows that the continuous cyclic motion brings the generated trajectories beyond the joint angle constrains, Eq. (27). This situation should be avoided because it can trigger to the failure of the manipulator structures. This is very contrast with the results from the proposed approach where the generated trajectory has satisfied the cyclic property and the posture continuity as shown in Fig. 13. The robot movements also have clearly been observed very efficient movement and smooth as has been observed in Fig. 7, 8, 11. 

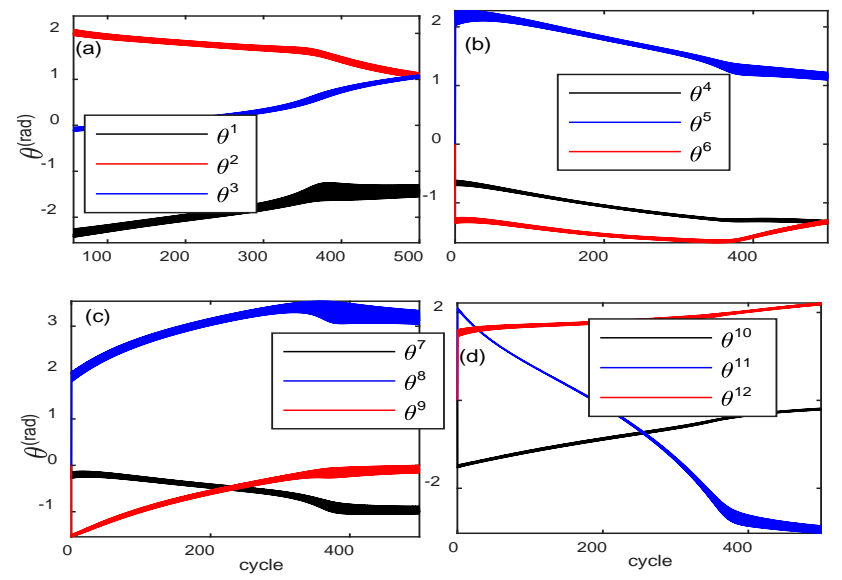

Fig. 12. Pseudoinverse Jacobian Generated Trajectories with 500-Cycle of Motion.
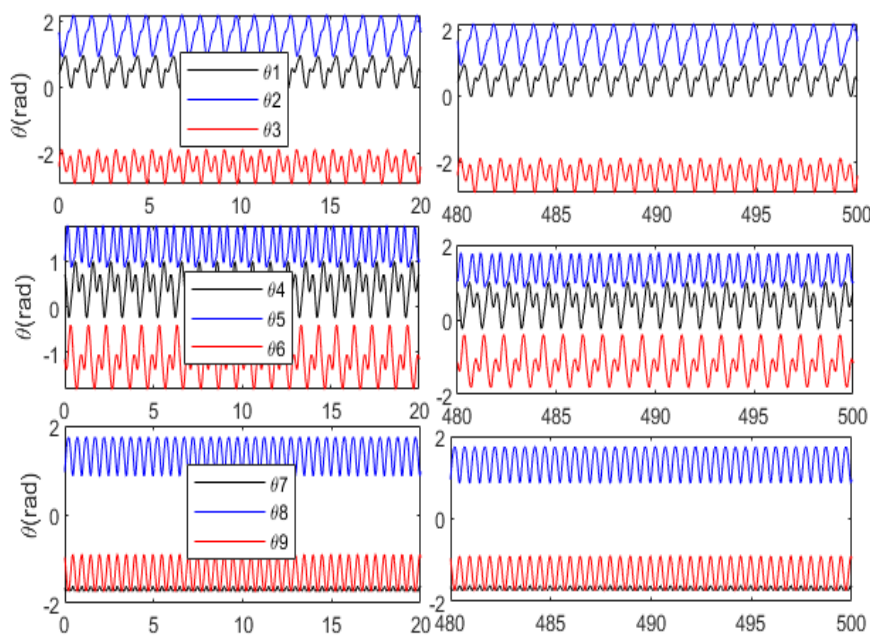

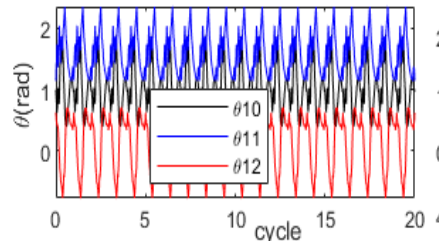

(a)

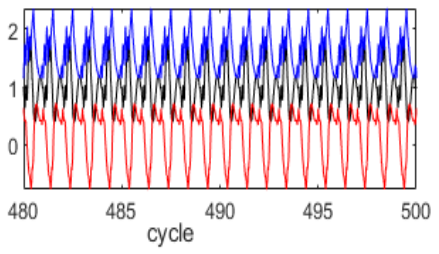

(b)
Fig. 13. Proposed Method with 500-Cycle of Motion (a) 20 Earliest Cyclic Motions (b) 20 Latest Cyclic Motions.

These results indicate that achieving the cyclic behavior is a challenging computational problem and it needs a computational strategy. The proposed method is an alternative method for achieving the cyclic property for the hyperredundant manipulator, instead of the Jacobian-based approach. The cyclic property is very essential because it guarantees the hyper-redundant motion lay within the safe zone, which is the operational area of the hyper-redundant manipulator.

Tracking the end-effector path by the hyper-redundant manipulator, there are many possible solutions. Using the Jacobian-based method, the results depend on the chosen initial configuration so that the exploration of the possible solutions to achieve the optimality is not possible. On the contrary, employing the meta-heuristic optimization, the possible solutions within the local orientation angle boundary have been explored and the optimal trajectories are founded successfully.

\section{CONCLUSION}

The computational approach to achieve the cyclic properties of the hyper-redundant manipulator based on the geometrical approach optimized by the meta-heuristic optimization approach had been presented. It showed that the redundancy resolution can be mapped into the boundary of the local orientation angle of the 3-link component. Since the hyper-redundant was composed from many links, there were the moving base. The moving base can be obtained through either the additional path if the previous 3-link was available or by generating periodic joint angle trajectories having the same period with the end-effector curve if the previous component was 2-link. Depending on the path planning case, several intermediate points may be necessary to achieve the good posture. The presentation of the obstacle yielded reducing the feasible area of the searching parameter. The meta-heuristic optimization has been employed to choose the best solution among many possible solutions of the inverse kinematics. As compared to the Jacobian based method, namely, Pseudoinverse Jacobian, the proposed path planning yields not only the cyclic motion but also the optimal path. In general, the WOA had the best performance than that of the GA where during 100 iterations, the WOA obtained the lowest fitness value. The proposed path planning involves finding the optimal local orientation angle trajectories. For the future research, generating the 3-link component trajectories which yield the better solutions than the constant function can be conducted to improve the performance of the developed approach.

\section{ACKNOWLEDGMENT}

The authors thank to Universitas Airlangga for supporting this research through SATU matching grant.

\section{AUTHOR's CONTRIBUTION}

A.M is the main contributor in this paper. S.P., A.A ${ }^{3}$, M.I.S., T.S.A.M., S.B., A.A ${ }^{7}$., N.W.R. are the supporting contributor.

\section{REFERENCES}

[1] M. Gadaleta, M. Pellicciari, and G. Berselli, "Optimization of the energy consumption of industrial robots for automatic code generation," Robot. Comput. Integr. Manuf. 57, (2019) 452-464.

[2] Y. Feng, Z. Ji, Y. Gao, H. Zheng, and J. Tan, "An energy-saving optimization method for cyclic pick-and- place tasks based on flexible joint configurations," of industrial robots for automatic code generation,” Robot. Comput. Integr. Manuf. 67, (2021), 102045, doi: 10.1016/j.rcim.2020.102037.

[3] L. Wang, A. Mohammed, X.V. Wang, and B. Schmidt, "Energyefficient robot applications towards sustainable manufacturing," Int. J. Comput. Integr. Manuf., 31 (8) (2018) 692-700.

[4] G. Chirikjian and J. Burdick, "A hyper-redundant manipulator," IEEE Robot. Autom. Mag. 1 (4), (1994) 22-29.

[5] K. A. Ayten, M. N. Sahinkaya, and A. Dumlu, Real time optimum trajectory generation for redundant/hyper-redundant serial industrial manipulators. Int. J. Adv. Robot. Syst. 14(6), (2017) 1-14.

[6] L. Sardana, M. K. Sutar, and P. M. Pathak, A geometric approach for inverse kinematics of a 4-link redundant in-vivo robot for biopsy. Robot. Auton. Syst. 61(12), (2013)1306-1313. 
[7] S. M. Menon, V. C Ravi, and A. Ghosal, Trajectory planning and obstacle avoidance for hyper-redundant serial robots. J. Mech. Robot. 9(4), (2017) 041010-1-041010-2.

[8] S. Yahya, M. Moghavvemi, and H. A. F. Mohamed, Geometrical approach of planar hyper-redundant manipulators: Inverse kinematics, path planning and workspace, Simul. Model. Pract. 19(1), (2011) 406422.

[9] H. Ananthanarayanan and R. Ordonez, A fast converging optimal technique to path planning hyper-redundant manipulator. Mech. Mach. Theory, 118 , (2017) 231-246.

[10] B. Dasgupta, A. Gupta, and E. Singla, A variational approach to path planning for hyper-redundant manipulators, Robot. Auton. Syst. 57(2), (2009) 194-201.

[11] A. Shukla, E. Singla, P. Wahi, and B Dasgupta, A direct variational method for planning monotonically optimal paths for redundant manipulators in constrained workspaces, Robot. Auton. Syst. 61(2), (2013) 209-220.

[12] E. Conkur, Path planning using potential fields for highly redundant manipulators. Robot. Auton. Syst. 52(2-3), (2005) 209-228.

[13] E. Lanteigne and A. Jnifene, Biologically inspired node generation algorithm for path planning of hyper-redundant manipulators using probabilistic roadmap. Int. J. Control Autom. 11, (2014). 153-161.

[14] G. S. Chirikjian and J. W. Burdick, The kinematics of hyper-redundant robot locomotion, IEEE Trans Robot Autom. 11(6), (1995) 781-93.
[15] G. S. Chirikjian and J. W. Burdick, A Geometric Approach to HyperRedundant Manipulator Obstacle Avoidance, ASME J. Mech. Des. 114(4), (1992) 580-585.

[16] S. Chiaverini, G. Oriolo, and I. Walker, Kinematically Redundant Manipulators. Siciliano, B. and Khatib, O. (Eds.), Springer Handbook of Robotics, Springer, New York, NY, (2008).

[17] M. daG. Marcos, J. A. T. Machado, and T. P. A. Perdicoúlis, An evolutionary approach for the motion planning of redundant and hyperredundant manipulators. Nonlinear Dyn. 60, (2010) 115-129

[18] M. daG. Marcos, J. A. T. Machado, J.A.T., and T. P. A. Perdicoúlis, A fractional approach for the motion planning of redundant and hyperredundant manipulators. Signal Process. 91(3), (2011) 562-570.

[19] J. A. T. Machado and A. M. Lopes, A fractional perspective on the trajectory control of redundant and hyper-redundant robot manipulators. Appl. Math. Model. 46, (2017) 716-726.

[20] S.K. Saha. Introduction to Robotics. Tata McGraw-Hill, New Delhi, India, (2008).

[21] A. Machmudah, S. Parman, A. Zainuddin, and S. Chacko, Polynomial joint angle arm robot motion planning in complex geometrical obstacles. Appl. Soft. Comput. 13(2), (2013) 1099-1109.

[22] S. Mirjalili, and A. Lewis, The whale optimization algorithm, Adv. Eng. Softw. 95, (2016) 51-67. 